\section{Ameloblastoma uniquístico intramural en maxilar superior. Reporte de un caso}

\author{
Intramural unicystic ameloblastoma in \\ upper jaw. A case report
}

\section{Resumen}

El ameloblastoma uniquístico (AU) representa aproximadamente el 6\% de todos los ameloblastomas y es frecuentemente encontrado durante la segunda década de la vida, sin predilección por sexo, se localiza en la mandíbula en relación a un diente no erupcionado, demuestra características clínicas y radiográficas similares a los quistes maxilares, pero evidencia histológicamente epitelio ameloblástico. Aunque el AU tiene un comportamiento menos agresivo que el ameloblastoma sólido, se recomienda un tratamiento radical para la variante intramural debido a su naturaleza infiltrativa, lo que tiende a potenciarse cuando se localiza en el maxilar superior. El objetivo del presente trabajo fue describir un caso de ameloblastoma uniquístico intramural localizado en el maxilar superior de un paciente masculino de 26 años de edad, quien presentaba un aumento de volumen indoloro en hemicara derecha de cinco meses de evolución. De acuerdo al resultado de la biopsia incisional se decidió realizar una hemimaxilectomía utilizando un abordaje intrabucal y colocación de una malla de titanio en piso de la órbita. La localización y el tipo histológico del AU son factores a considerar en la elección de la terapéutica, es importante el seguimiento a largo plazo del paciente para valorar la posibilidad de recidivas y un abordaje interdisciplinario del caso.

Palabras clave: Ameloblastoma; Maxila; Neoplasias orales; Tumores odontogénicos.

\section{Caso Clínico}

Mariangela Victoria Valecillos Fuenmayor ${ }^{1, a}$, Gerardo José Guillen Rivera 2,a, Ana Isabel Ortega-Pertuz ${ }^{1, a}$, Ligia Pérez ${ }^{1, a}$

${ }^{1}$ Universidad del Zulia, Facultad de Odontología. Maracaibo, Venezuela.

2 Oral Maxillofacial and Implant Surgery Center. Texas, USA.

a Doctor en Odontología.

\section{Correspondencia:}

Mariangela V. Valecillos Fuenmayor

Correo electrónico: mariangelavalecillos@ gmail.com

Universidad del Zulia, Facultad de Odontología, Departamento de Medina Bucal, Cátedra de Cirugía Bucal. Avenida 19 con calle 65. Maracaibo, Venezuela.

\section{Coautores:}

Gerardo José Guillen Rivera geraldguillenr@gmail.com Ana Isabel Ortega-Pertuz anitaortegav@gmail.com Ligia Pérez ligiaperezcastro@cantv.net

Conflicto de intereses: Los autores declaran no tener conflictos de interés.

Fuente de financiamiento: Autofinanciado

Fecha de recepción: 25/05/18

Fecha de aceptación: 06/08/18

\begin{abstract}
Unicystic Ameloblastoma (UA) accounts for approximately 6\% of all ameloblastomas. They are often observed during the second decade of life, without a predilection for gender, with $90 \%$ of the lesions occurring in the jaw and related to an unerupted tooth. Unicystic ameloblastoma shows clinical and radiographic features similar to maxillary cysts but histologically evidences ameloblastic epithelium. The lesion has a less aggressive biological behavior than solid ameloblastoma, with a lower recurrence rate compared with conservative treatment, however, a radical therapeutic is recommended for the intramural variant. The aim of the present work was to describe a case of UA located in the maxilla of a 26-year-old male patient, who showed an increase in volume in the right hemiface of five months of evolution. The histopathological examination of the specimen obtained by the incisional biopsy was UA Intramural; In a second surgical tempo, a hemimaxilectomy was performed on the affected side using an intra-oral approach. Long-term follow-up of these lesions is recommended to assess the possibility of relapse and an interdisciplinary therapeutic-rehabilitation approach.
\end{abstract}

Keywords: Ameloblastoma; Maxilla; Odontogenic tumor; Oral neoplasms. 


\section{Introducción}

El ameloblastoma uniquístico (AU) fue descrito por primera vez en 1977 por Robinson y Martínez ${ }^{1}$ como una entidad clínica distinta al ameloblastoma sólido por mostrar un aspecto histológico y morfológico particular, representando aproximadamente entre el $6-28 \%$ de todos los ameloblastomas ${ }^{2,3}$. Habitualmente los AU están asociados a un órgano dental no erupcionado. La presencia de un diente no erupcionado intralesional permite diferenciar dos variantes: AU dentígero y no dentígero. El 90\% de las lesiones han sido reportadas en el sector posterior de la mandíbula, seguido de la parasinfisis, región anterior y posterior del maxilar 1,3,4-6. En la mayoría de los casos, el AU se presenta en pacientes jóvenes durante la segunda década de la vida 2,7-10. Cuando el tumor está relacionado a un diente no erupcionado, el AU muestra una ligera predilección por el sexo femenino $(1,6: 1)^{9}$.

Usualmente el AU comienza como una lesión ósea central asintomática, que progresiva y lentamente tiende a extenderse en vez de perforar el hueso. A medida que aumenta de tamaño puede provocar migración, desviación y movilidad dentaria y alteraciones en la oclusión. De igual manera puede evidenciarse fallos en la secuencia y cronología de la erupción en los dientes adyacentes. La erosión de la mucosa raramente se produce a pesar de la expansión de las corticales ${ }^{3,4,6,10-12}$.

La patogénesis del AU es incierta. Las teorías de que el AU se origina "de novo" como una neoplasia propiamente dicha o bien, que es el resultado de la transformación neoplásica del epitelio de una lesión quística preexistente y más específicamente a partir del quiste dentígero son aún discutibles, siendo probable que ambos mecanismos ocurran ${ }^{1,13}$. Leider et al. ${ }^{14}$ propusieron tres mecanismos patogénicos para la formación del AU: 1) Que derive directamente del órgano del esmalte; 2) Que una forma sólida degenere o se transforme en quística; 3) Que se produzca una transformación ameloblástica en los quistes odontogénicos y/o ameloblastomas asociados a folículos de dientes incluidos después de los 30 años de edad.

Ackerman et al. ${ }^{13}$ clasifican el AU en tres subtipos histológicos: AU I (luminal), AU II (intraluminal) y AU III (mural), de acuerdo a las características presentes en el epitelio de la lesión. Por su parte, Philipsen y Reichart 2 describen cuatro subgrupos histológicos: subgrupo 1: AU luminal, subgrupo 1,2: AU luminal e intraluminal, subgrupo 1,2,3: AU luminal, intraluminal e intramural y subgrupo 1,3: AU luminal e intramural.

Radiográficamente la lesión se presenta como un proceso osteolítico con localización pericoronal, interradicular o periapical ${ }^{9}$, en general muestra bordes bien definidos, en un patrón uni o multilocular, siendo el de mayor ocurrencia el unilocular ${ }^{1,3,4,6,7,13,15}$, este último suele estar asociado con dientes retenidos de pacientes jóvenes con una edad media de 22 años, mientras que el tipo multilocular se encuentra en adultos con una edad media de 33 años ${ }^{16,17}$. En estados avanzados puede evidenciarse adelgazamiento y expansión de las corticales, así como reabsorción radicular ${ }^{1,2,13,15}$.

La terapéutica quirúrgica del $\mathrm{AU}$ contempla desde el manejo conservador con enucleación, curetaje acompañado de marzupialización de la lesión, hasta la hemisección del maxilar afectado ${ }^{7,18}$. En este sentido existe un consenso general en el que tanto el tipo histológico luminal como el intraluminal pueden ser tratados mediante enucleación, mientras que los AU intramurales, por su capacidad de infiltración, deben ser considerados como AM sólidos ${ }^{2,16,19}$. La tasa de recurrencia del AU después de un tratamiento quirúrgico conservador ocupa entre el 10 al 25\% de los casos reportados, esta cifra es menor que el 50 al 90\% de la tasa de recurrencia observada después del curetaje del AM sólido ${ }^{9}$. Este trabajo describe un caso clínico de ameloblastoma uniquístico intramural (AUIM) con localización infrecuente en región canina del maxilar superior, destacando los aspectos clínicos, imagenológicos, histológicos y quirúrgicos.

\section{Reporte del caso}

Paciente masculino de 26 ańos de edad acude a la consulta por presentar un aumento de volumen en la hemicara derecha de aproximadamente cinco meses de evolución. Durante la anamnesis, se descartan antecedentes de trauma local, así como antecedentes personales y familiares relevantes. Como dato afirmativo al interrogatorio, el paciente refiere dolor localizado de intensidad leve, espontáneo durante la palpación en la zona afectada. Los exámenes de laboratorio complementarios mostraron parámetros normales:

Hematología: hemoglobina: 12 gr/L, hematocrito: $39,0 \%$, tiempo de protrombina (TP): 13 ", tiempo de tromboplastina (TPT): 30", plaquetas: $270000 \mathrm{~mm}^{3}$, leucocitos: $5600 \mathrm{~mm}^{3}$. Bioquímica Sanguínea:

Glicemia: $90 \mathrm{mg} / \mathrm{dL}$, urea: $27 \mathrm{mg} / \mathrm{dL}$, creatinina: 0,8 $\mathrm{mg} / \mathrm{dL}$.

Serología: V.D.R.L: no Reactivo, HIV: negativo.

$\mathrm{Al}$ examen extrabucal, se observó un aumento de volumen difuso en la región geniana de la hemicara derecha, con ligera elevación del ala nasal y acentuación del surco nasoyugal. La piel de la zona se apreció de aspecto y coloración normal, sin cambios de temperatura (Figura 1A-C).

El examen intrabucal reveló un aumento de volumen bien circunscrito de consistencia firme y de aproximadamente $3 \mathrm{~cm}$ de diámetro ubicada en la región canina derecha. La mucosa que recubre la lesión se evidenció adelgazada y de color rojo intenso (Figura 1D-E). Se ve- 
rificó la ausencia de las piezas dentarias 11, 46 y 48; así como múltiples caries en los dientes remanentes.

En la imagen panorámica se evidenció una imagen radiopaca, que se extendía desde la pieza dentaria 12 hasta el 17, con aspecto de lesión ocupante de espacio del seno maxilar ipsilateral (Figura 2), que causaba divergencia de las raíces de las piezas dentarias 14 y 15 , así como reabsorción radicular externa de los ápices de las piezas dentarias 12,14 y 15 . En virtud de las características clínicas de la lesión, el tiempo de evolución y localización, la impresión diagnóstica inicial fue de 1)
Tumor odontogénico quístico queratinizante, 2) Mixoma odontogénico y 3) Ameloblastoma.

De acuerdo a los diagnósticos diferenciales considerados, el plan de tratamiento fue una biopsia incisional y el análisis histopatológico. El informe microscópico señaló que las secciones mostraron una cavidad quística revestida por epitelio escamoso estratificado delgado con áreas de mayor espesor, en otras con superficie glandular, presencia de microquistes y células mucosas. Se observó zonas de efecto inductivo, además de células en empalizada de las células basales y polaridad reversa
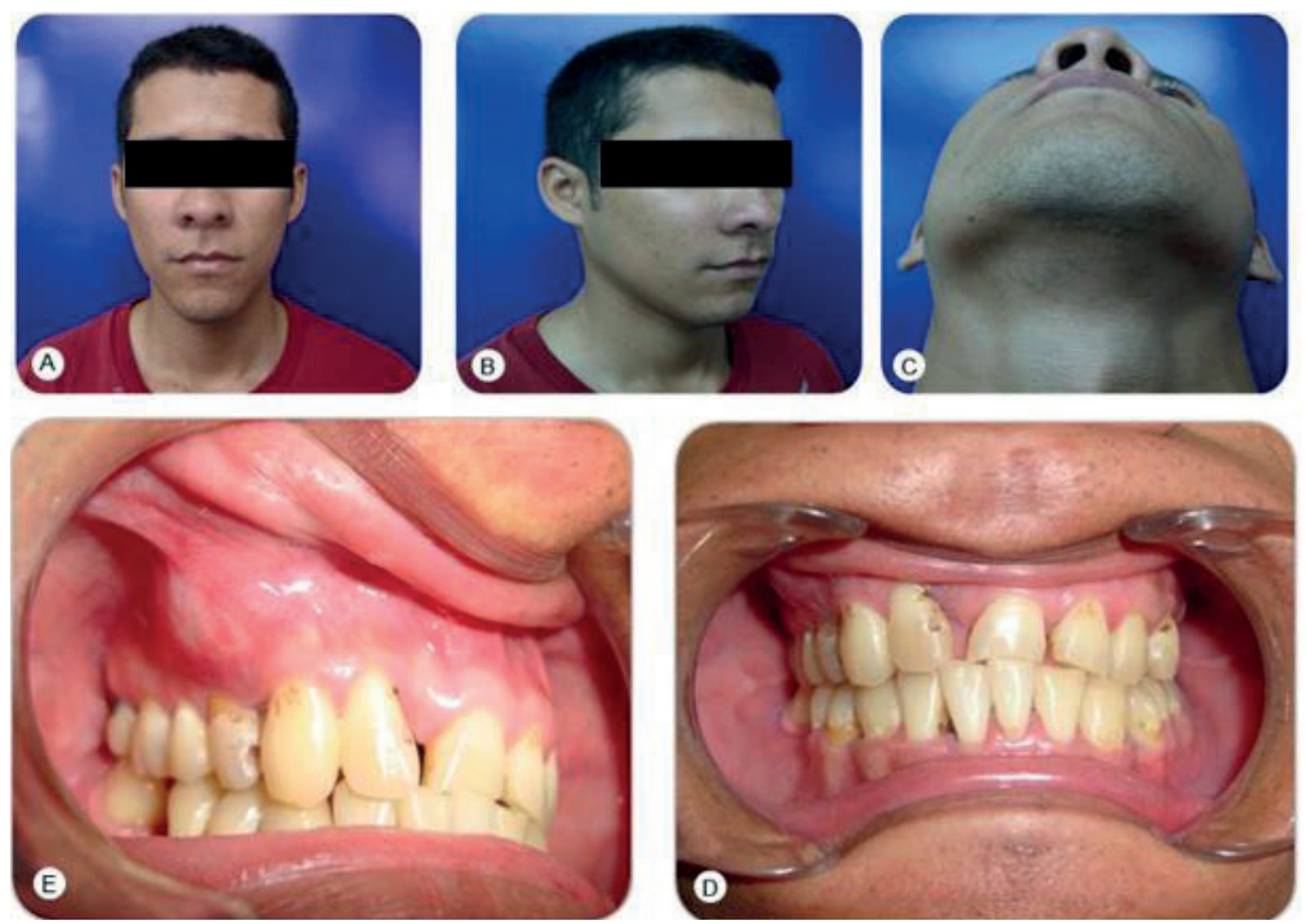

Figura 1. A. Vista frontal extrabucal, B. Vista lateral derecha, C. Vista inferior del paciente donde se evidencia aumento de volumen en región geniana de la hemiarcara derecha. D y E. Vista intrabucal de la lesión tumefacta recubierta de mucosa de coloración rojo intenso

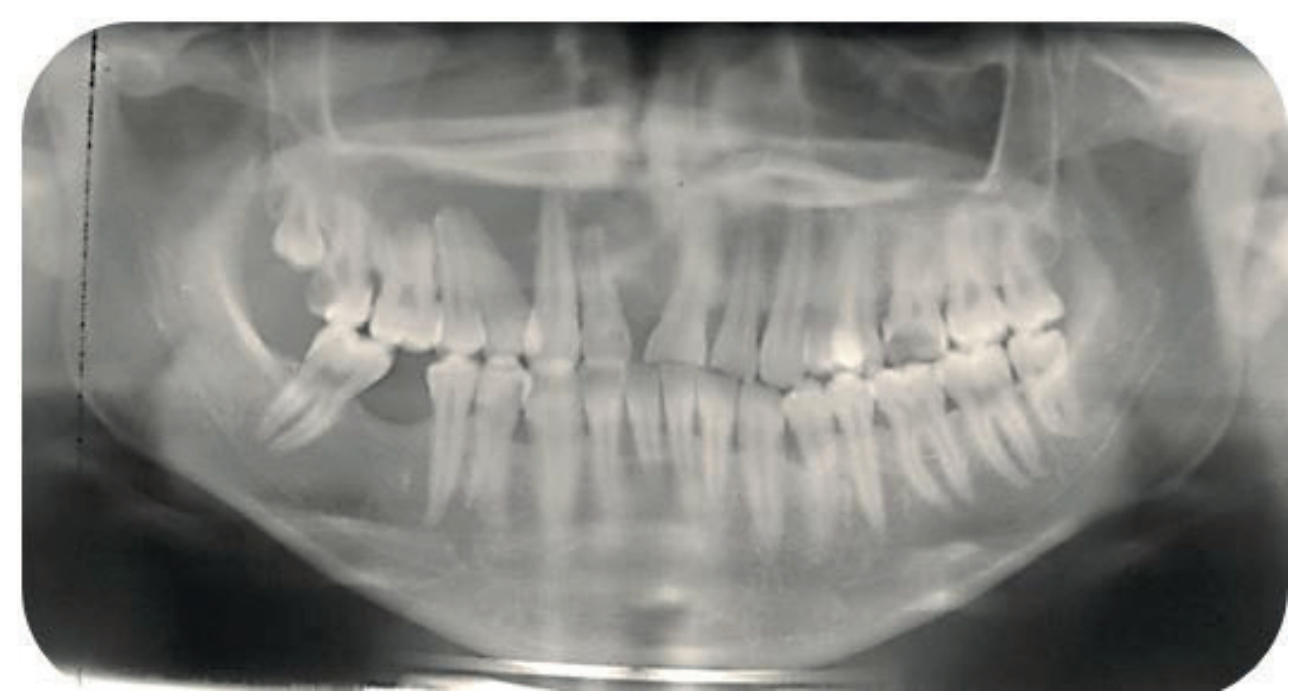

Figura 2. Radiografía panorámica inicial muestra opacificación del seno maxilar derecho 


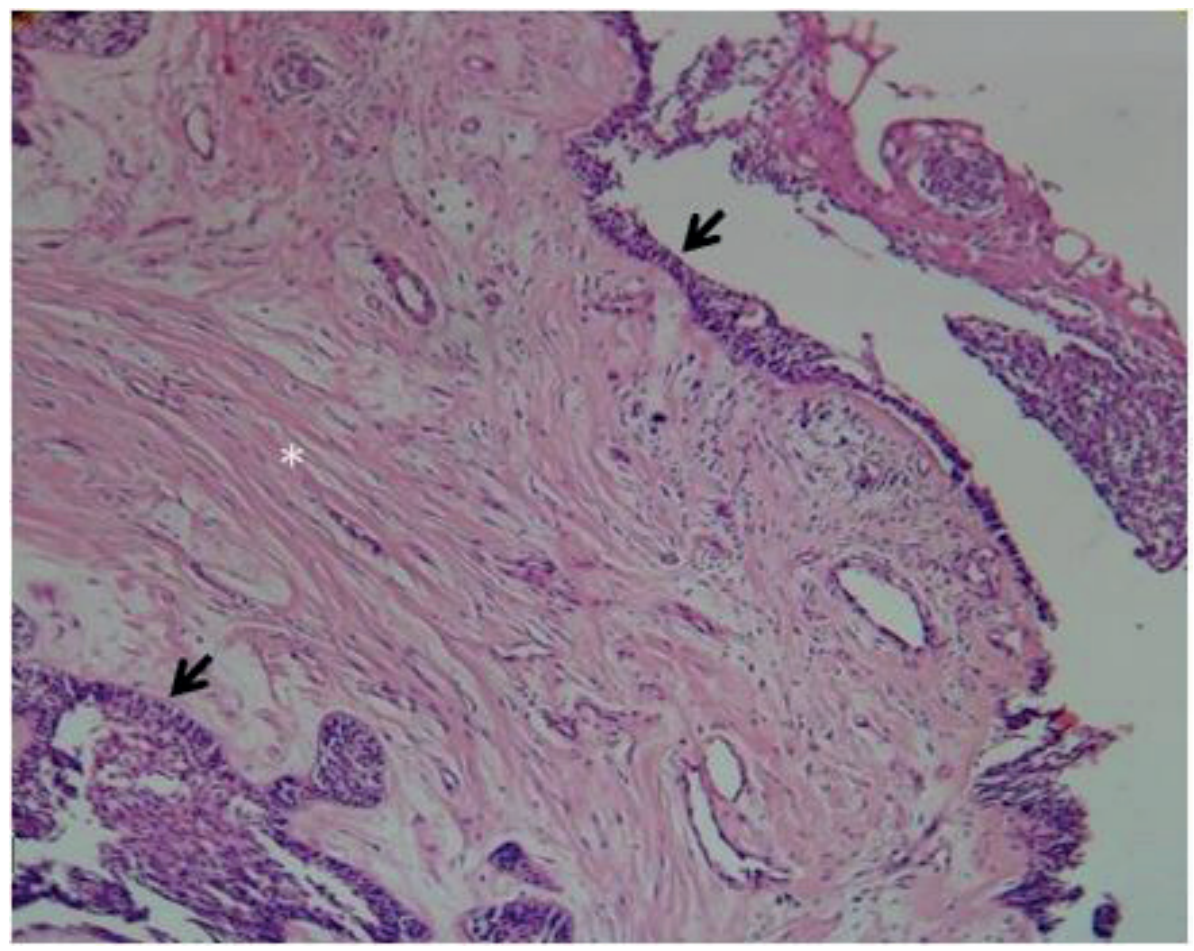

Figura 3. Microfotografía 100x con H-E que evidencia presencia de epitelio de origen ameloblástico (cabezas de flechas) soportado en un estroma celular denso con áreas de fibrosis (asterisco)

(Figura 3). En otras zonas, las células epiteliales se organizaban laxas entre sí, imitando el retículo estrellado del órgano del esmalte de un diente en desarrollo. El resto de la pared estaba constituida por tejido conectivo fibroso, que contenía islas de células epiteliales organizadas en la misma forma descrita. Se observaron trabéculas de tejido óseo maduro vital y un leve infiltrado inflamatorio mononuclear. Estos hallazgos histopatológicos fueron compatibles con el diagnóstico de AUIM.

Se indica la realización de una tomografía computarizada para el análisis y planificación quirúrgica del caso. En asociación de reconstrucciones multiplanares y cortes axiales, se observó una imagen isodensa sugestiva de lesión ocupante de espacio, localizada en el seno maxilar derecho que causaba erosión del piso de órbita y se extendía al complejo osteomeatal derecho. Además, se evidenció compromiso de la porción horizontal del hueso maxilar ipsilateral (Figura 4).

Posteriormente se programó la resección del tumor y hemimaxilectomía, la toma de muestra para biopsia excisional y su correspondiente análisis histopatológico. Bajo anestesia general balanceada, con intubación orotraqueal se realizó abordaje bucal con incisión envolvente en el maxilar y una descarga anterior en zona de la pieza dentaria correspondiente a la 23 y en la zona posterior a nivel de la tuberosidad. Se procedió al levantamiento del colgajo mucoperióstico, incorporando en los márgenes de resección, porciones de tejido blando que se encontraban en contacto con hueso erosionado (Figura 5A-B). Se realizó la exodoncia de la pieza dentaria 12, lo cual constituyó el margen anterior de la resección del tumor. A nivel posterior, el margen se extendió hasta la tuberosidad del maxilar y zona pterigopalatina.
En sentido cefálico caudal, se realizó osteotomía en el contrafuerte maxilo-malar incorporando en la pieza quirúrgica la porción del piso de orbita, razón por la cual se realizó un abordaje extrabucal, mediante incisión subciliar (Figura 5C-E), para la colocación de material de osteosíntesis, consistente en malla de titanio en piso de la órbita, fijada en el reborde orbitario con tornillos del sistema 1.5. Finalmente, se realizó la síntesis de los tejidos con sutura reabsorbible, alcanzando afrontar los márgenes del lecho quirúrgico y estableciendo separación anatomo-funcional entre cavidad bucal, seno maxilar y fosas nasales. Durante el control clínico postoperatorio a una semana de la intervención quirúrgica, se apreció discreto edema facial, palpebral y decoloración subciliar del lado derecho y leve sensibilidad dolorosa. Intraoralmente se apreció la herida con una evolución favorable sin evidencia de comunicación bucosinusal. El examen histológico de la pieza quirúrgica, ratificó el diagnóstico histológico inicial de AUIM. A los cuatro años de la cirugía, el paciente continúa sin signos clínicos o radiográficos de recidiva.

\section{Discusión}

El AU es un tumor poco frecuente que se presenta aproximadamente en el 6\% de los casos de AM ${ }^{2,3}$. Usualmente afecta a pacientes jóvenes en edades comprendidas entre los 16 y 26 años, el 50\% de las lesiones ocurren en la segunda década de la vida, tal y como es en el caso presentado. En cuanto a la localización, más del 90\% de los casos se han observado en el sector posterior de la mandíbula, su presencia en la maxila es rara ${ }^{2,8,9,10,20}$. El paciente del presente reporte mostraba un compromiso del maxilar que se extendía de la porción anterior al interior del seno maxilar ipsilateral. 

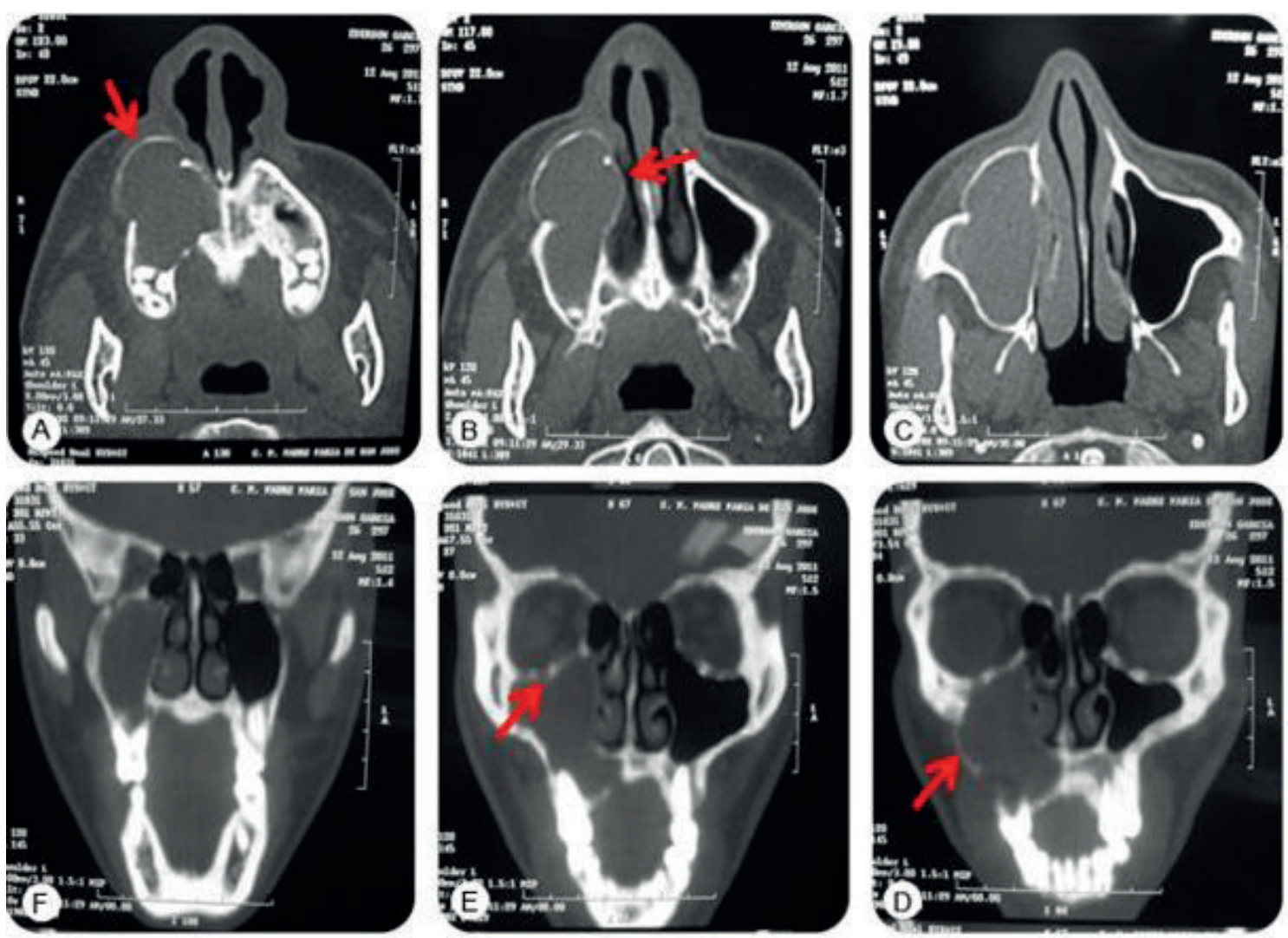

Figura 4. Examen de tomografía computarizada. A-C. Cortes axiales que evidencian opacificación del seno maxilar derecho, expansión de la pared anterior y medial del mismo (flechas). D-F. Vistas coronales en los que se observa la ocupación del seno maxilar y reborde alveolar de la maxila así como el compromiso del piso de orbita (flechas)
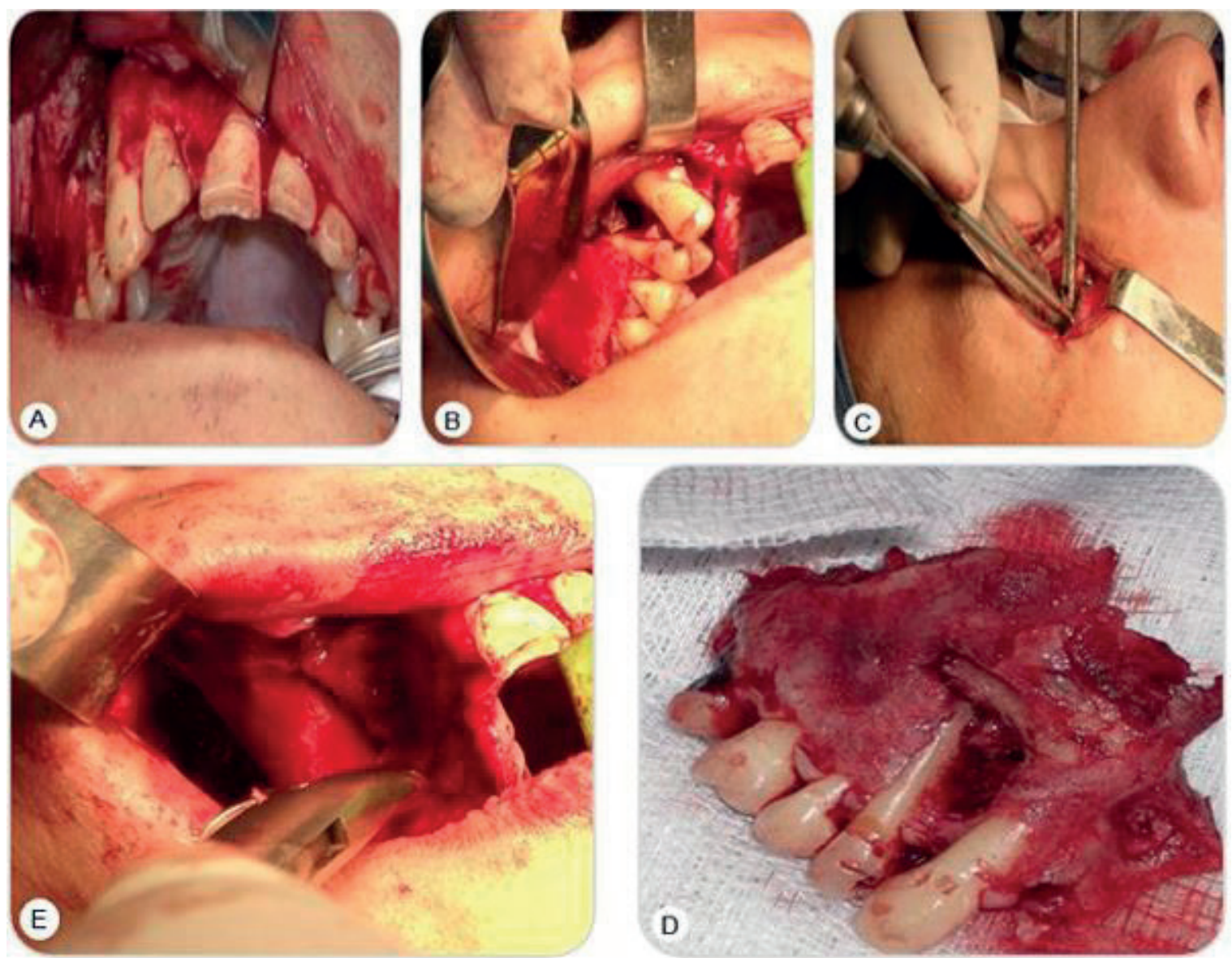

Figura 5. A y B. Abordaje intrabucal con levantamiento de colgajo mucoperióstico incorporando los márgenes de la lesión. C. Abordaje subciliar para la colocación de material de osteosíntesis. D. Pieza quirúrgica. E. Vista intrabucal del defecto óseo 
El AU suele poseer un patrón de crecimiento lento y silente, sin originar síntomas en sus estadios más precoces. Las lesiones de gran tamaño, cursan con asimetría facial indolora que pueden provocar migración, desviación, movilidad de piezas dentarias, reabsorción radicular e incluso parestesia. La ulceración de la mucosa rara vez se presenta, aunque las lesiones en estadios avanzados pueden expandir las corticales o las erosiona invadiendo el tejido blando 17,20. Las manifestaciones clínicas del caso mostrado difieren con las que usualmente se reportan, debido a que el paciente a pesar del aparente poco tiempo de evolución de la asimetría facial, refiere dolor a la palpación, de intensidad leve y aparición espontánea, lo que posiblemente esté relacionado con la localización del tumor en la maxila con ocupación del seno maxilar respectivo.

Los hallazgos clínicos y radiográficos en la mayoría de los casos de AU son sugestivos de lesiones quísticas odontogénicas como el quiste dentígero, asociadas a dientes no erupcionados; en estos, casi en su totalidad estaría involucrado un tercer molar mandibular no erupcionado. Las lesiones de $\mathrm{AU}$ que no están relacionados a alguna estructura dentaria retenida se les denomina variante no dentígera como en el caso presentado ${ }^{4,6,18}$.

Radiográficamente el AU se observa como un proceso osteolítico con bordes bien definidos y esclerosados. Las presentación radiográfica del AU unilocular es más frecuente que el patrón multilocular, los dientes involucrados en la lesión pueden mostrar grados variables de reabsorción radicular y en estadios avanzados movilidad dentaria ${ }^{6,7,13,15}$. En el caso estudiado se observó tanto la reabsorción apical en premolares e incisivo lateral como migración y desviación de los dientes adyacentes a la lesión.

El diagnóstico histopatológico sólo puede ser realizado cuando la presencia de epitelio ameloblastomatoso es identificado inequívocamente en estas lesiones ${ }^{23}$. Estos cambios ameloblásticos, podrán observarse en toda la extensión de la membrana quística o sólo focalmente 2,13. La extensión mural dentro de la pared quística es un hallazgo frecuentemente registrado ${ }^{2}$. El término AUIM es usado cuando el revestimiento bien sea plexiforme o folicular penetra el tejido capsular ayacente ${ }^{2,9}$. Por otro lado, Gunawardhana et al. ${ }^{23}$ señalan que algunas características histológicas pueden ser útiles en la diferenciación histológica de AU de los quistes odontogénicos: a) presencia de cambios mixomatosos en la pared del quiste, b) proliferaciones similares a epitelio prexiforme en la ausencia de inflamación, c) morfología de mamelones alargados con la ausencia de inflamación, c) morfología de mamelones alargados con hialinizacion subepitelial. De acuerdo a las características presentadas, el caso coincide con un AU mural o Grupo III, puesto que el análisis histopatológico de la biopsia incisional refiere una cavidad quística revestida por epitelio escamoso estratificado delgado en unas áreas, mayor espesor en otras con superficie glandular, presencia de microquistes y células mucosas; así como también zonas de efecto inductivo, además de células basales en empalizada y polaridad reversa. De igual manera se evidenció que en otras áreas las células epiteliales se organizan laxas entre sí, imitando el retículo estrellado del órgano del esmalte de un diente en desarrollo. Si bien, en algunas ocasiones la biopsia incisional no es representativa de la totalidad de la lesión, esto pudiese generar errores en la correcta clasificación de las mismas. La pared epitelial de los AU intramurales se caracteriza por no ser siempre uniforme y a menudo la lesión está rodeada por un delgado epitelio no específico que imita la pared de un quiste dentígero. De manera que la certeza histopatológica se obtiene realizando una biopsia excisional ${ }^{9}$. No obstante, en el caso presentado, se logró obtener un diagnóstico inequívoco con el análisis histopatológico de la muestra obtenida de la biopsia incisional que fue confirmado con los hallazgos histopatológicos del análisis del espécimen obtenido en la biopsia excisional.

Desde el punto de vista del diagnóstico diferencial, el AU carece de características patognomónicas que permitan identificarlo clínicamente y diferenciarlo fácilmente del resto de las lesiones odontogénicas. Si se consideran la edad, localización y las características clínico-radiográficas, el diagnóstico diferencial se limita a entidades en tres categorías: tumores odontogénicos, lesiones quísticas y otras lesiones benignas no odontogénicas ${ }^{17,21}$. De la variedad de tumores odontogénicos habría que considerar aquellas lesiones radiolúcidas expansivas que pueden estar asociadas o no a dientes retenidos y/o desplazamiento de dientes adyacentes, movilidad, divergencia de raíces y extrusión de dientes erupcionados como el tumor odontogénico epitelial calcificante o tumor de Pindborg en su variante radiolúcida, mixoma odontogénico, así como también el tumor odontogénico quístico queratinizante y otras variantes histológicas del ameloblastoma ${ }^{22}$.

La planificación del tratamiento para el AU depende del tipo histológico. Los AU del subtipo luminal e intraluminal pueden ser abordados de manera conservadora y efectivamente mediante enucleación, mientras que el subtipo intramural o subtipos 1,2,3 y 1,3 de Philipsen y Reichart ${ }^{2}$ por su capacidad de infiltración deben considerarse como un ameloblastoma sólido y por lo tanto tratados de forma radical ${ }^{2,9}$. Asimismo, las lesiones maxilares, como en el presente caso, son consideradas más agresivas, con peor pronóstico y por presentar más dificultades en cuanto al tratamiento, lo que tuvo influencia en el tratamiento de elección ${ }^{24}$. En el maxilar el hueso cortical es más delgado que en la mandíbula y no es capaz de confinar el crecimiento tumoral. La combinación de un hueso fino y frágil con la proximidad del maxilar a la cavidad nasal, senos paranasales, órbita y otras estructuras vitales en la base del cráneo agregan una dimensión clínica no presente en los tumores mandibulares. Para las localizaciones maxilares, sobre todo las inherentes a los sectores lateroposteriores, se indican en primera instancia, cirugías más agresivas, al considerar la capacidad de desarrollarse de forma asintomática y poder infiltrar el seno maxilar, la fosa pterigopalatina, la fosa temporal y la base craneal ${ }^{21,24}$. 
Los defectos de los maxilares luego de la resección de un tumor conducen frecuentemente a la pérdida en la eficacia de las funciones propias del sistema estomatognático y a una severa deformidad estética. Para aumentar la calidad de vida de estos pacientes se recomiendan idealmente, sistemas de injertos óseos y rehabilitación implantólogica de acuerdo a las posibilidades socio-económicas de los mismos.

En conclusión, el manejo del AU debe ser guiado por su comportamiento biológico; por lo tanto, la naturaleza infiltrativa del AUIM indica un tratamiento radical. Las lesiones maxilares suelen ser más agresivas por la configuración del tejido óseo, siendo esta localización infrecuente para el AUIM. En estos casos es importante el seguimiento clínico y radiográfico continuado para descartar cualquier tipo de recidiva que se podría presentar.

\section{Referencias bibliográficas}

1. Robinson L, Martínez MG. Unicystic ameloblastoma: a prognostically distinct entity. Cancer. 1977;40(5):227885.

2. Philipsen HP y Reichart PA. Unicystic ameloblastoma. A review of 193 cases from the literature. Oral Oncol. 1998;34(5):317-25.

3. Fregnani ER, da Cruz Perez DE, de Almeida OP, Kowalski LP, Soares FA, de Abreu Alves F. Clinicopathological study and treatment outcomes of 121 cases of ameloblastomas. Int J Oral Maxillofac Surg. 2010;39(2):145-9.

4. Gupta N, Saxena S, Rathod VC, Aggarval P. Unicystic ameloblastoma of the mandible. J Oral Maxillofac $\mathrm{Pa}$ thol. 2011;15(2):228-31.

5. De Melo WM, Pereira-Santos D, Sonoda CK, Pereira-Freitas SA, de Moura WL, de Paulo Cravinhos JC. Large unicystic ameloblastoma of the mandible: management guided by biological behavior. J Craniofac Surg. 2012;23(5):e499-502.

6. Kumar KR, George GB, Padiyath S, Rupak S. Mural unicystic ameloblastoma crossing the midline. A rare case report. Int J Odontostomat. 2012;6(4):97-103.

7. Seintou A, Martinelli-Kla CP, Lombardi T. Unicystic ameloblastoma in children: systematic review of clinicopathological features and treatment outcomes. Int J Oral Maxillofac Surg. 2014;43(4):405-12.

8. Artés Martínez MJ, Prieto Rodríguez M, Navarro Hervás M, Peñas Pardo L, Camańas Sanz A, Vaquero de la Hermosa $\mathrm{MC}$, et al. Diagnóstico citológico de las recidivas tumorales de ameloblastoma: Presentación de dos casos clínicos. Med Oral Patol Oral Cir. 2005;10(3):205-9.

9. Nagalaxmi V, Sangmesh M, Naik K, Kodaga S, Chappidi V, Goyal S. Unicistic mural ameloblastoma: An unusual case report. Case Rep Dent. [Internet]. 2013 [citado el 10 de marzo de 2018]; Disponible en: https://www.hindawi.com/journals/crid/2013/957418/

10. Valarezo J. Importancia del diagnóstico histopatológico de los ameloblastomas previo a la cirugía radical con reconstrucción mandibular. [tesis de bachiller]. [Guayaquil]. Facultad Piloto de Odontología, Universidad de Guayaquil; 2012: 45p.

11. Castillo L, González de Palmero MC, Tinoco P. Ameloblastoma uniquístico. Presentación de un caso clínico. Acta Odontol Venez. 1997;35(3):20-25.

12. García NG, Oliveira DT, Rodríguez MTV. Unicystic ameloblastoma with mural proliferation managed by conservative treatment. Case Rep Pathol. [Internet] 2016 [citado el 10 de marzo de 2018]; Disponible en: https://www.ncbi.nlm.nih.gov/pmc/articles/ PMC5005522/

13. Ackerman GL, Altini M, Shear M. The unicystic ameloblastoma. A clinicopathologic study of 57 cases. J Oral Pathol Med. 1998;17(2):541-6.

14. Leider AS, Eversole LR, Barkin ME. Cystic ameloblatoma. A clinicopathologic analysis. Oral Surg Oral Med Oral Pathol.1985;60(6):624-30.

15. Hsu MH, Chiang ML, Chen JK. Unicystic ameloblastoma. J Dent Sci. 2014;9:407-11.

16. Argandoña J, Espinoza Y. Ameloblastoma uniquístico, bases del tratamiento conservador. Presentación de caso clínico y actualización de la bibliografía. Rev Esp Cir Oral Maxilofac. 2011;33(2):88-92.

17. Beovide AV. Ameloblastoma Uniquístico - La Importancia de un Diagnóstico Histológico. Universidad de la República. Facultad de Odontología. Cátedra de Anatomía Patológica. Montevideo, Uruguay. [Consultado el 10 de diciembre de 2017]. Disponible en: http://conganat. uninet.edu/IVCVHAP/PDF/C004.pdf

18. Lau SL, Samman N. Recurrence related to treatment modalities of unicystic ameloblastoma: A systematic review. Int J Oral MaxilloFac Surg. 2006;35(8):681-90.

19. Vagas G, Liceaga R, Trujillo JJ, Liceaga C. Tratamiento de los ameloblastomas. Análisis de 26 casos. Rev Mex Cir Bucal Max. 2010;6(2):66-72.

20. Rebolledo M, Manotas I, Harris J. Parámetros diagnósticos del ameloblastoma maxilar: Reporte de caso inusual en un niño. Rev CES Odontol. 2011;24(1):37-41.

21. Morales D. Ameloblastoma. Revisión de la literatura. Artículo de revisión. Rev Cubana Estomatol. 2009;43(3):48-61.

22. Kalaskar R, Unawane AS, Kalaskar AR, Pandilwar P. Conservative management of unicystic ameloblastoma in a young child: Report of two cases. Contemp Clin Dent. 2011;2(4):359-63.

23. Gunawardhana KS, Jayasooriya PR, Tilakaratne WM. Diagnostic dilemma of unicystic ameloblastoma: novel parameters to differentiate unicystic ameloblastoma from common odontogenic cysts. J Investig Clin Dent. 2014;5(3):220-5.

24. Parmar S, Al-Qamachi L, Aga H. Ameloblastomas of the mandible and maxilla. Curr Opin Otolaryngol Head Neck Surg. 2016;24(2):148-54. 